Bull. Austral. Math. Soc.

$90 \mathrm{~B} 99,05 \mathrm{C} 05,52 \mathrm{~A} 45$

VOL. 37 (1988) [473-475]

\title{
EXISTENCE THEOREMS FOR FLOORPLANS
}

\section{RINSMA}

The existence of planar floorplans, composed of non-overlapping rooms divided from each other by walls, to suit given topological and dimensional constraints cannot. always be guaranteed. Only a finite number of arrangements exist for the layout of rooms on a single floor with specified adjacencies between them, regardless of their shape or size. Limits on the geometry of the plan and dimensional constraints reduce this variety of arrangement further.

The use of computers for automated floorplan design using either heuristics or exhaustive methods has concentrated on rectangular rooms and perimeters. Graph theoretic approaches have been used over the past twenty years. A recent comprehensive survey is in Steadman (1983).

In the case of area constraints the equations to be solved for a particular rectangular floorplan can be found (Earl and March (1979)) but a solution may not always satisfy the adjacency requirements. On the other hand, very few of the toplogically feasible floorplans may yield feasible solutions in the dimensioning step. It seems that, at this point in the investigation, it was unknown whether or not a rectangular floorplan could always be found to satisfy both given adjacency and area requirements.

The thesis studies this problem by providing theorems for the existence of floorplans with given area and adjacency constraints. It concentrates on plans in which each room is adjacent to the exterior, and on three types of floorplans - rectangular, isometric and convex.

The adjacency requirements between rooms are represented in a graph called the weak dual. If each room is external, the graph must be outerplanar (Lynes (1977)), and the allowable graphs range from trees to maximal outerplanar graphs.

Proper floorplans have weak duals which are maximal outerplanar. Weal duals of proper rectangular floorplans have at most four vertices of degree 2 , but proper isometric and convex floorplans have no such restriction.

Received 14 August 1987

Thesis submitted to University of Canterbury January 1987. Degree approved in May 1987. Supervisor: Dr. D.F. Robinson

Copyright Clearance Centre, Inc. Serial-fee code: 0004-9729/88 \$A2.00+0.00. 
Given a maximal outerplanar graph with at most four vertices of degree 2 and areas for each vertex or room it is investigated whether a proper rectangular floorplan can be found having the given graph as its weak dual and satisfying the area requirements.

This involves colouring the graph and then drawing the corresponding nonisomorphic dimensionless floorplans. By exploiting the existence of a fault line in a proper rectangular floorplan, each nonisomorphic floorplan can be drawn directly from each "different" coloured graph. Each floorplan so formed has area conditions that need to be satisfied if the plan is to be dimensioned to suit particular areas for the rooms.

By considering these plans and conditions the thesis shows a proper rectangular floorplan can always be found satisfying both area and adjacency requirements given by a weak dual $G$ that is maximal outerplanar with two or three vertices of degree 2 . However a solution cannot be guaranteed if $G$ has four vertices of degree 2 .

It is then shown that allowing through rooms in rectangular floorplans is often more restrictive.

Chapter V shows that proper isometric floorplans cannot, while proper convex can, always exist satisfying area conditions and having any maximal outerplanar graph for its weak dual.

Chapter VI shows that if the given adjacencies and areas can be represented in a tree, then any of the three types of floorplans can always be found satisfying all the constraints.

The tree in this case is a spanning tree of the floorplan's weak dual. This leads to an interesting question in graph theory - what restrictions exist on the embedding of a tree in a maximal outerplanar graph? This is answered in Chapter III. The branching index is defined and its properties examined. From this, another index, $\varepsilon$, is used to give the minimum number of vertices of degree 2 of any maximal outerplanar graph in which a given tree can be embedded. An algorithm details a particular embedding for which this minimum number is achieved.

Chapter VII uses these results. Here the given adjacencies and areas are represented by a tree. A floorplan is to be found in which each room is external and the given constraints are satisfied.

It is shown that a proper rectangular floorplan can always be found providing the embedding index of the tree is 0 or 1 . If it is 2 , then the only maximal outerplanar graphs in which the given tree can be embedded may be those for which no plan satisfying both area and adjacency conditions are possible. However it is shown an exterior rectangular floorplan in this case is possible. If the index is greater than two, no exterior rectangular floorplan is possible.

Under the same area and adjacency constraints, a proper isometric floorplan (with each room external) cannot always be guaranteed. A proper convex floorplan, however, 
is always possible.

The addendum extends the investigation to non-convex rooms. An orthogonal floorplan in which each room is a rectangle or L-shaped can always be found satisfying any of the three types of adjacency and area conditions considered in the main body of the thesis.

The thesis ends with a review of the study, along with possible applications and extensions for future research.

\section{REFERENCES}

[1] C.F. Earl and L.J. March, 'Architectural applications of graph theory', Applications of Graph Theory (1979), 327-355.

[2] J.A. Lynes, 'Windows and floor plans', Environment and Planning B 4, 51-56.

[3] J.P. Steadman, Architectural Morphology (Pion, London, 1983).

Department of Mathematics and Statistics

Massey University

Private Bag

Paluerston North

New Zealand 